\section{Paediatric SLE}

\section{LUPUS NEPHRITIS IN CHILDREN: A 7 YEAR SINGLE CENTRE EXPERIENCE FROM INDIA}

M Agarwal*, A Shivpuri, S Sawhney. Sir Ganga Ram Hospital, Paeditric Rheumatology, New Delhi, India

\subsection{6/lupus-2017-000215.124}

Background and aims There is no class I evidence available to treat children with lupus nephritis (LN). This study looked at our experience of management of $\mathrm{LN}$ and contribute to the existing world literature. In addition to treatment of $\mathrm{LN}$, care is given to educate the family, manage lipids, BMI, restricting steroid use to $1 \mathrm{mg} / \mathrm{kg} / \mathrm{day}$ at onset, give hydroxychloroquine to all and vaccinate if possible.

- To study the clinical profile and lab parameters of children at onset of nephritis.

- To see which of the two drugs cyclophosphamide(CYC) or mycophenolate mofetil (MMF)were more effective by studying the time to renal flare.

- To analyse the side effects and disease related damage in these children

Methods All children with lupus nephritis who attended the Paediatric Rheumatology clinic from Sept 2009 to Sept 2016 were included.

Results 166 children with SLE, 67 had LN 67/166 (40.3\%); Male: Female=1: 2.72. Median SLEDAI at nephritis onset:18 57 renal biopsies:Class I:1, Class II:5, Class III:19, Class IV:26, Class V:6 MMF used to induce remission:43(64\%), Cyclophosphamide (CYC) 19 (28\%) Azathioprine:5(7\%). 67\% achieved complete remission during induction. 25\% partial remission/flared after an initial response within induction period. Median time to response during induction therapy: 4 months (2-17 months). MMF was given to $82 \%$ and Azathioprine to $18 \%$ for maintenance. $36 / 62$ (58\%) never flared, 23/ $62(37 \%)$ flared during induction therapy and 3/62 (5\%) were in partial remission.
Abstract 124 Table 2 Outcome variables

\begin{tabular}{|l|l}
\hline Variable & \\
\hline Medianduration of induction therapy & 7 months $(3-35)$ \\
\hline Median duration of follow up since nephritis & 48 months(3-159) \\
diagnosis & \\
\hline MedianSLEDAI at onset of nephntis & $18(4-52)$ \\
\hline MedianSLEDAI at last follow up & $0(0-43)$ \\
& \\
\hline At last follow up: & \\
Complete remission & $58 \%$ \\
Active ds & $9 \%$ \\
Disease flare & $7 \%$ \\
Complete remission off DMARDs & $4 \%$ \\
Lost to follow up & $16 \%$ \\
Deaths & $4 \%$ \\
& \\
& \\
\hline Infectionprofile & \\
Cellulitis & $4 \%$ \\
Tuberculosis & $3 \%$ \\
Enteric fever & $3 \%$ \\
Viral infections: & $34 \%(23 / 67)$ \\
Herpes zoster & $40 \%(9 / 23)$ \\
Dengue & $40 \%(9 / 23)$ \\
CMV reactivation & $13 \%(3 / 23)$ \\
Varicella & $4 \%(1 / 23)$ \\
Adeno vinus & $4 \%(1 / 23)$ \\
& \\
\hline
\end{tabular}

The primary outcome measure, time to renal flare was statistically insignificant regardless of the induction agent used Conclusions MMF and CYC were equally effective as induction agents and neither was superior to prevent renal flares. No factor: demographic, clinical or laboratory could predict renal flares. $58 \%$ were in renal remission, $33 \%$ on steroids.

Abstract 124 Table 1 Basic demographics

\begin{tabular}{|l|l|}
\hline Median & \\
\hline Age at onset of SLE disease & llyears( 4-18years) \\
\hline Age at diagnosis of SLE disease & 11.75 years(4.5-18.16years) \\
\hline Delay to diagnosis & $2.76 \mathrm{months}(0.24-72)$ \\
\hline Age at nephritis diagnosis & 12.2 years( $4.5-24.5$ years) \\
\hline $\begin{array}{l}\text { Time to onset of nephritis (46 children had simultaneous onset } \\
\text { of SLE disease and nephritis)21 children had a delayed onset }\end{array}$ & 19 months((6.72-120 months) \\
& \\
\hline Sr. Creatinine at onset of induction & $0.7 \mathrm{mg} / \mathrm{dl}(0.27-2.84)$ \\
\hline Sr.Creatinine at onset of maintenance & $0.42 \mathrm{mg} / \mathrm{dl}(0.16-3)$ \\
\hline Unine spot protein creatinine ratio at onset of induction & $1.96(0.35-13.19)$ \\
\hline Urine spot protein creatinine ratio at onset of maintenance & $1.3 \mathrm{gm}(0.240-5.5)$ \\
\hline
\end{tabular}

